Voix et Images

volxetimages

\title{
Georges-Étienne Cartier : un objet de sémiologie théâtrale
}

\section{Bernard Andrès}

Volume 4, numéro 2, décembre 1978

Guy Lafond

URI : https://id.erudit.org/iderudit/200163ar

DOI : https://doi.org/10.7202/200163ar

Aller au sommaire du numéro

Éditeur(s)

Les Presses de l'Université du Québec

ISSN

0318-9201 (imprimé)

1705-933X (numérique)

Découvrir la revue

Citer cet article

Andrès, B. (1978). Georges-Étienne Cartier : un objet de sémiologie théâtrale.

Voix et Images, 4(2), 337-341. https://doi.org/10.7202/200163ar d'utilisation que vous pouvez consulter en ligne.

https://apropos.erudit.org/fr/usagers/politique-dutilisation/ 


\section{Georges-Étienne Cartier : un objet de sémiologie théâtrale}

Georges-Étienne Cartier ou la Prise par surprise est un montage de textes historiques présenté par Jean-Guy Sabourin au théâtre de la Grande Réplique, en octobre 1978. Ce spectacle est intéressant à divers titres. II s'attache à réconcilier le Québec et son Histoire, dans l'esprit des Grands Soleils de Ferron et plus récemment, Du Dernier Recours de Baptiste à 


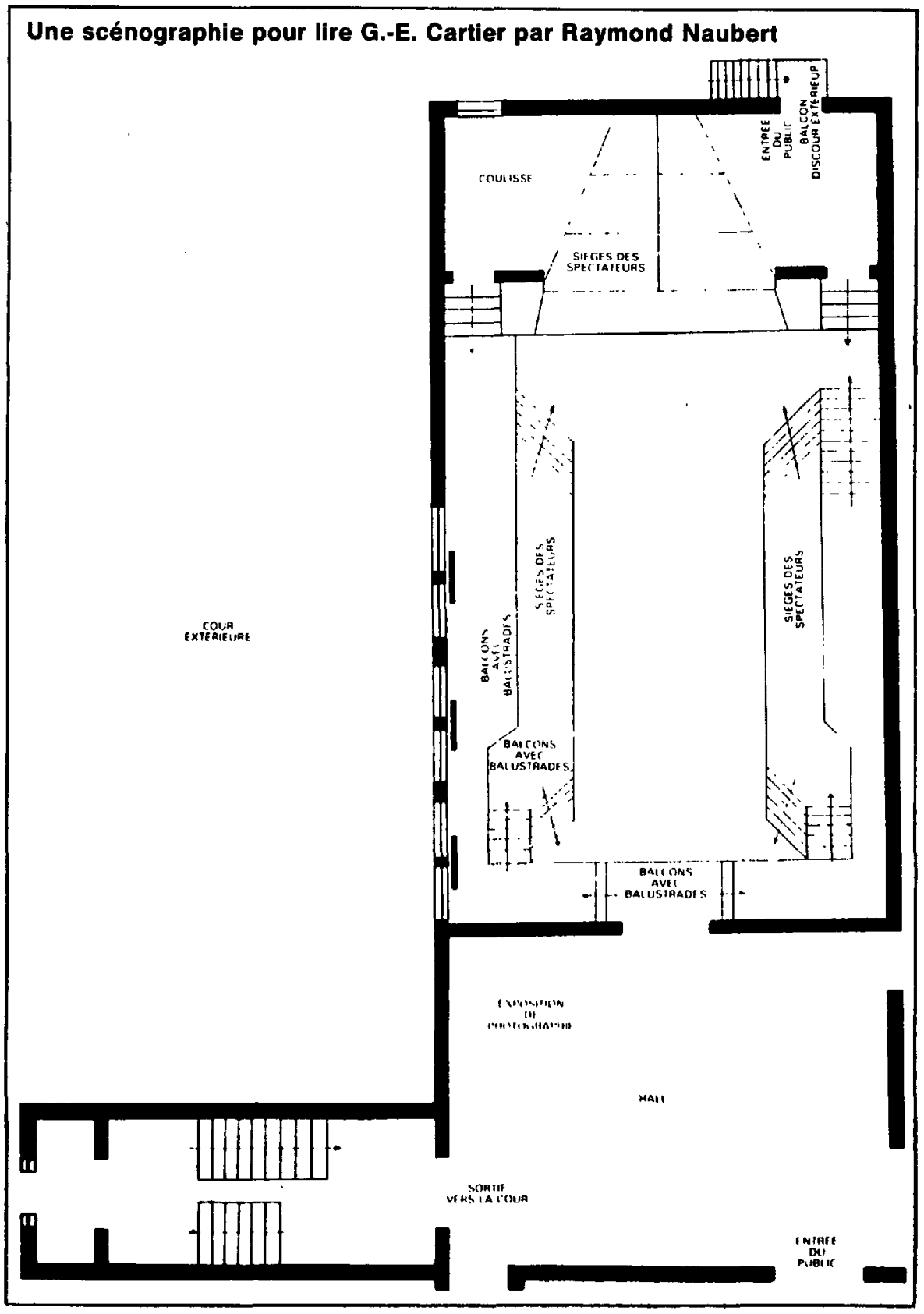


Catherine, de Michèle Lalonde. II confirme également l'orientation esthétique et politique d'un nouveau groupe théâtral. «Terre-Québec, théâtre de la Grande Réplique " est né voilà deux ans d'une expérience douloureuse. On se rappelle la controverse soulevée alors par la pièce politique Chili Vencera, montée au T.P.Q. Mais Georges-Étienne Cartier s'impose d'abord à la critique pour deux raisons : $1^{\circ}$ ce montage scénique marie une structure filmique et un système théâtral à partir de textes historiques et $2^{\circ}$ il est jouable. D'une part, la prouesse technique qui consiste à mêler presque inextricablement le tournage d'un film sur la Confédération canadienne, et la théâtralisation (réelle) de ce tournage (fictif). D'autre part, l'intérêt d'un spectacle vivant où le spectateur a autre chose à faire qu'à consommer placidement un spectacle.

Signalons qu'au moment d'écrire ces lignes, il est question que l'ensemble soit filmé pour de bon. Cela permettra au sémiologue de la représentation de se faire la dent sur un cas précieux d'interaction de deux codes sémiotiques dont les effets contradictoires ou redondants modulent sur le même référent (la confédération), toute une gamme de signifiés (de l'exaltation de cette période historique à sa condamnation sans recours). Entre les deux extrêmes, la graduation des nuances conservatrices et libérales, par rapport auxquelles doit se situer un spectateur attentif et participant. L'argument est simple :

Nous sommes en octobre 1978 et nous préparons un vidéo pour les

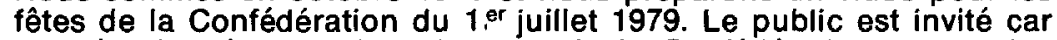
le scénario nécessite la naissance de la Confédération et certains événements connus ou méconnus de 1864 à $1872^{1}$.

Sur ce canevas, Jean-Guy Sabourin construit un système dont la complexité peut être évoquée à partir de l'analyse des personnages. Je me bornerai ici à un rapide survol de ces catégories actorielles dans la plèce.

Les «acteurs" sont des personnages de plein droit: leur présence sur le plateau ne vaut pas seulement pour le film; elle définit au second degré le statut du comédien (dans le rôle de l'acteur). Celui-ci n'est que le signifiant filmique du signifié théâtral. Ce système de relais pourrait être explicité comme suit : quand le «réalisateur " demande à l' "acteur" de reprendre tel discours de Cartier, il commande au comédien de rejouer l'emploi de l'acteur dans le rôle du personnage historique de G. E. Cartier. Le même procédé est utilisé pour le «caméraman ", les «musiciens" et le "commentateur" (qui fait la voix off). Les «reporters" qui interrogent «le spectateur" (encore un rôle, nous le verrons) à l'entrée, jouent de même sur deux niveaux. En interviewant le public au sujet de la Confédération, ils agissent en figurants pour le film, et en comédiens pour la représentation. Si l'on considère à présent les personnages «principaux», ce n'est pas vers Cartier, Dorion ou $\mathrm{M}^{{ }^{g}}$ Bourget qu'il faut se tourner, mais bien vers le trio formé par le réalisateur, régisseur et la recherchiste. 
Le «Réalisateur (J.-G. Sabourin) fait double office. II incarne un conflit de niveaux de représentation des plus intéressants : coordonateur fictif et présent du film, et concepteur réel et absent de la pièce. C'est lui qui, au cœur d'une envolée lyrique de Cartier, jette: "Coupez!", pour enchaîner sur la séquence suivante... ou conseiller à l'acteur de reprendre sur un autre ton! Le même discours prend alors une autre allure, voire une signification nouvelle (autant de voix et de voies entre lesquelles je dois choisir pour construire ma propre vision des choses). C'est le soir de la première que le rôle du réalisateur, ou plutôt sa fonction sémique dans le spectacle, apparut dans toute son ambiguïté (au sens étymologique). En présence du recteur de I'UQAM, le sous-ministre de la Culture, lui aussi invité d'honneur, prononça un discours sur la mission de l'historien au Québec. Le ton était simple, l'allocution courte, mais cela n'allait pas sans créer une certaine gêne, du fait même de la présence des autres comédiens sur le plateau. Alors que tout le dispositif scénographique disait que le spectacle théâtral était amorcé (même si l'on ne «tournait " pas encore le vidéo fictif), un spectateur (fut-il sous-ministre), venait interrompre le jeu pour parler (suprême enchassement!)... de la carrière de J.-G. Sabourin, concepteur du spectacle. Réaction de Sabourin-réalisateur : il embraya immédiatement après le discours sur: “En place pour la séquence 2 " (enchâssant à son tour le discours du sous-ministre dans le texte du spectacle).

La “recherchiste» a le rôle le plus ingrat: celle qui semble la plus discrète dans la machine cinématographique (en principe, elle n'apparaît simplement pas), se trouve représentée dans ce tournage. Il lui incombe en outre d'introduire tous les personnages historiques en répondant à la question du réalisateur: "Qu'est-ce qu'on a sur $X, Y$, ou $Z$ ?" Sur ses épaules repose en fait l'arsenal didactique de la représentation. Sous prétexte d'offrir à l'acteur les données historiques susceptibles de le guider dans son interprétation de Dorion, Mgr Bourget ou Médéric Lanctôt, elle fournit au spectateur des compléments d'information sur la genèse de la Confédération. Il va sans dire que la ficelle est grosse et que, sans l'humour de la comédienne et les interventions du réalisateur, le procédé tomberait à plat.

La recherchiste : «Tocqueville est un grand historien français [...] Sa rigueur scientifique lui confère encore aujourd'hui le respect de ses pairs. "

Le Réalisateur : "Bon ça va, on y va."

Le couple recherchiste-réalisateur définit à lui seul l'orientation «progressiste" du spectacle, tant au niveau formel qu'idéologique. Après le discours de Cartier aux élections de 1867, le réalisateur en demande un autre de Médéric Lanctôt, principal opposant dans Montréal-Est. 
La recherchiste: “M. Lanctôt ne fait pas trois lignes dans les histoires du Canada; mais j'ai un manifeste de lui sur la condition ouvrière...”

Le réalisateur: «Donne ce texte à $M$., peut-être que lu lentement sans trop d'effets de voix, ça pourra passer. "

Suit l'extrait du manifeste, ponctué par ces remarques qui, tout en cautionnant Lanctôt, modalisent ses propos et posent tout le problème de l'esthétique réaliste :

Le Réalisateur: "C'est bon, mais on ne peut pas tourner cela, le monde va croire qu'on l'a fabriqué avec les idées de notre temps. "

Un acteur: "Faut pas prendre le monde pour des fous. »

Le réalisateur: [...] «En art, c'est le vraisemblable qui est vrai. La véritè de ce texte est telle qu'elle a l'air fausse [sic]... "

Ces considérations sur le type de décodage effectué par le spectateur, place ce dernier au centre de la problématique. Comment ne pas prendre "le monde pour des fous", si l'on pousse par ailleurs le public à prendre parti? Invité comme figurant (= acteur) dans le film à tourner, il est avant tout le spectateur du théâtre de la Grande Réplique ${ }^{2}$. Mais à trop se prendre au jeu (en participant aux élections de 1872, par exemple), il se surprend lui-même comme comédien... Et l'on se prend à l'interroger sur la polysémie possible du sous-titre: “... ou la prise par surprise». Du Canadien français de 1867 ou du Québécois de 1978, lequel des deux s'est le mieux laissé prendre?

Bernard Andrès

1. On trouve le texte de Georges-Étienne Cartier dans le volume 2, $\mathrm{n}^{\circ} 1$ des $\mathrm{Ca}$ hiers de la Grande Réplique, 1406, rue Saint-Denis, Montréal, Québec. Soulignons l'intérêt de ces cahiers qui d'un spectacle à l'autre, proposent une réflexion critique sur les pièces et sur des problèmes théoriques de dramaturgie contemporaine.

2. Le schéma scénographique ci-joint donne une idée de la configuration spatiale de la pièce : le spectateur occupe fictivement la place des députés au Parlement. 